\title{
Particles and Strings in Degenerate Metric Spaces
}

\author{
Luís A. Cabral and Victor O. Rivelles \\ Instituto de Física, Universidade de São Paulo, \\ Caixa Postal 66315, 05315-970 São Paulo, SP, Brazil \\ e-mail: lacabral@fma.if.usp.br, rivelles@fma.if.usp.br
}

\begin{abstract}
We consider relativistic and non-relativistic particles and strings in spaces (or space-times) with a degenerate metric. We show that the resulting dynamics is described by a rich structure of constraints. All constraints are classified and the dynamics depends strongly on the parity of the difference between the dimension of the space (or space-time) and the rank of the degenerate metric. For a particular class of degenerate metrics we can identify the null eigenvectors of the metric with its Killing vectors. We also give the first steps towards the quantization of the non-relativistic particle using the Senjanovic path integral quantization and the Batalin-Fradkin-Tyutin conversion method.
\end{abstract}

Keywords: Degenerate Metrics, Constrained Systems, String Theory, Path Integral Quantization, BFT Method

PACS: 03.65.Ca, 11.10.Ef, 11.25.-w

Typeset using REVTEX 


\section{INTRODUCTION}

Singularities have a fundamental role in general relativity [1]. However it is not clear how to incorporate them into a quantum gravity theory. Surely singularities are an important feature in quantum gravity as demonstrated by processes like Hawking radiation. However our inability to follow the black hole evaporation process till its very end shows our present limitations. In string theory the situation is somehow ameliorated. The entropy of extreme and near-extreme black holes, for instance, can be computed by considering D-branes located near the horizon [2]. At weak coupling these D-branes are described by a supersymmetric gauge theory for which it is possible to count the relevant states for the entropy. Since the D-branes are located near the horizon the singularities inside the black hole have not yet been properly treated in string theory. There are, however, some attempts in this direction [3.

The singularities which give rise to black holes manifest themselves in the curvature tensor. The effect of this sort of singularity on the propagation of quantum particles has recently been studied [4]. There is however another sort of singularity for which the curvature tensor itself is not singular. They appear when the metric tensor is degenerate and therefore has no inverse. If the degeneracy occurs on a set of measure zero then the curvature remains bounded and the topology of the space-time manifold can change [5]. This sort of singularity is also compatible with the causal structure of space-time [6]. Such singularities are milder than curvature singularities and perhaps it should be easier to handle them in a quantum gravity theory.

In general relativity degenerate metric appears in the Palatini formulation. There we start with an action in terms of a tetrad $e_{\mu}^{a}$ and a Lorentz connection $\omega_{\mu}^{a b}$. The action is $S=\frac{1}{2} \int e^{a} e^{b} R^{c d} \epsilon_{a b c d}$ where $e^{a}$ is the tetrad one-form and $R=d \omega+\omega^{2}$ is the curvature twoform. The space-time metric is then $g_{\mu \nu}=e_{\mu}^{a} e_{\nu}^{b} \eta_{a b}$ where $\eta_{a b}$ is the tangent space Minkowski metric. This action and the equations of motion which follow from it do not depend on the inverse tetrad so they are well defined even when the tetrad $e_{\mu}^{a}$ is degenerate. Solutions describing degenerate tetrads have been found and a relation between them and solutions of two-dimensional BF theories has been established [7]. In a less fundamental level degenerate metrics also appear as induced metrics on null hypersurfaces [8]. In the four dimensional case the induced degenerate metric is of rank two.

Degenerate metrics or tetrads have also appeared in other contexts. Ashtekar formulation of general relativity employs as phase space variables a complex potential $A_{a i}$ and its electric field $E^{a i}$ in a complexified $\mathrm{SO}(3)$ Yang-Mills theory [9]. The usual metric interpretation of general relativity requires that the spatial metric be proportional to $E^{a i} E^{b i}$. Ashtekar formalism remains well defined even when $E^{a i}$ is degenerate. Solutions presenting boundaries in which one of the sides has a degenerate metric have been found [10]. Also many gravity theories in two and three dimensions can be formulated as a gauge theory of the ChernSimons type or BF type [11]. All of them are valid when the zweibein or dreibein are degenerate. It has also been suggested that there would be a topological phase of quantum gravity in which the tetrad vanishes and diffeomorphism invariance is unbroken 12.

In the context of string theory it has been shown that strings can propagate in degenerate metric backgrounds. When there are only right or left movers the metric has a degeneracy

of rank two [13. It is also known that p-branes which behave as instantons are described 
by a degenerate metric [14]. Also, tensionless strings [15] and tensionless D-branes [16] are governed by a dynamics that involves a degenerate metric.

All this indicates that degenerate metrics may have an important role in quantum gravity. In fact, if we intend to incorporate degenerate metrics in a quantum theory of gravity we should first understand its classical effects. Some work has already been done in this direction. At the classical level the main consequence of the degenerate metrics is to allow topology change of the space-time manifold [5]. Since this is not observed in nature there must exist a suppression mechanism for it, probably at the quantum level. On the other side the behavior of matter in space-times with a degenerate metric have not been properly explored. In the case of topological field theories the metric appears nowhere in the action so that the coupling to matter is trivial. Only global issues are important in this case. However, when we consider matter fields with canonical kinetic terms the coupling to gravity requires the introduction of contravariant tensors fields. The simplest example is the action of the scalar field which depends on the inverse metric. To treat these cases an algorithm has been proposed which does not need any contravariant tensor fields [17]. However there is another important class of matter which can be coupled to the metric tensor but does not require contravariant tensor fields. It is constituted by particles and strings. The action and field equations of particles and strings do not depend on the inverse metric and are well defined even when the metric becomes degenerate.

In this paper we will consider the dynamics of particles and strings in spaces or spacetimes with a degenerate metric. We will analyze the structure of the constraints imposed by the degenerate metric and find the effective degrees of freedom which describe the motion of particles and strings. We will first consider the non-relativistic particle in Section [I]. A complete analysis of the constraints will be done. It will be developed in detail since the constraint analysis of the subsequent sections will be very close to that of the nonrelativistic particle. In Section III we will study the relativistic particle and in Section $\mathbb{1}$ strings will be considered. In Section $\nabla$ we will analyze the role of the isometries of degenerate metrics and its relation to the analysis done in the former sections. We will also consider a preliminary quantization of the non-relativistic particle in Section V1. There we will use the path integral quantization due to Senjanovic [18 and also the Abelian conversion method of Batalin-Fradkin-Tyutin (BFT) [19]. Finally Section VII presents some remarks and conclusions.

\section{NON-RELATIVISTIC PARTICLE}

Usually we interpret a degenerate metric space of dimension $D$ and rank $r$ as a lower dimensional space with dimension $r$. However we wish to consider a degenerate metric space in a broader sense. To implement this consider a set of $D$ vectors in the lower dimensional space $e_{i}^{a}, i=1, \ldots, D, a=1, \ldots, r$. From them we can build a degenerate metric $g_{i j}=$ $e_{i}^{a} e_{j}^{b} h_{a b}$, where $h_{a b}$ is an Euclidean metric, in such a way that $g_{i j}$ has rank $r$. Indeed, if we consider a set of vectors $U_{\alpha}$ in the $\mathrm{D}$ dimensional space then the equation $g_{i j} U_{\alpha}^{j}=0$, viewed as a linear system, has $D-r$ solutions for $U_{\alpha}$ since the rank of $g_{i j}$ is $r$. These are the null eigenvectors of the metric. Notice that only in the case that $e_{i}^{a}$ is locally integrable, that is, if $e_{i}^{a}=\frac{\partial y^{a}}{\partial x^{i}}$, then it describes in fact a $r$ dimensional space.

Let us now consider a particle of mass $m$ moving in such a space. Its Lagrangian is 


$$
L=\frac{1}{2} m g_{i j} \dot{x}^{i} \dot{x}^{j}=\frac{1}{2} m \dot{x}^{a} \dot{x}^{b} h_{a b}
$$

where $\dot{x}^{a}=e_{i}^{a} \dot{x}^{i}$. The canonical momentum is

$$
P_{i}=\frac{\partial L}{\partial \dot{x}^{i}}=m g_{i j} \dot{x}^{j}=m e_{i}^{a} \dot{x}_{a}
$$

where $\dot{x}_{a}=h_{a b} \dot{x}^{b}$. Because the metric is degenerate we can not invert this equation to find the velocity $\dot{x}^{i}$ in terms of the momentum $P_{i}$. This means that the system has constraints. If we consider the null eigenvectors $U_{\alpha}$ introduced above we find that

$$
P_{i} U_{\alpha}^{i}=m g_{i j} \dot{x}^{j} U_{\alpha}^{i}=0
$$

so that there are $D-r$ primary constraints

$$
\phi_{\alpha}=P_{i} U_{\alpha}^{i}, \quad \alpha=r+1, \ldots, D .
$$

The range of the indice $\alpha$ was chosen in this way for future convenience.

To find an explicit expression for $U_{\alpha}$ we consider, without loss of generality, the first $r$ components of Eq.(2). They can be solved for the $r$ unknowns $\dot{x}^{a}$ as

$$
\dot{x}_{a}=\frac{1}{m} \tilde{e}_{a}^{i^{\prime}} P_{i^{\prime}}, \quad i^{\prime}=1, \ldots, r,
$$

where $\tilde{e}_{a}^{i^{\prime}}$ is the inverse of $\tilde{e}_{i^{\prime}}^{a}$. Notice that $\tilde{e}_{i^{\prime}}^{a}$ is a square matrix build up from the first $r \times r$ components of the original $e_{i}^{a}$. The $D-r$ remaining equations in Eq.(2) are then

$$
P_{\alpha}=m e_{\alpha}^{a} \dot{x}_{a}=e_{\alpha}^{a} \tilde{e}_{a}^{i^{\prime}} P_{i^{\prime}}
$$

which give rise to the constraints found in Eq.(四)

$$
\phi_{\alpha}=P_{\alpha}-e_{\alpha}^{a} \tilde{e}_{a}^{i^{\prime}} P_{i^{\prime}}
$$

The explicit expression for $U_{\alpha}$ is then

$$
U_{\alpha}^{i}=\delta_{\alpha}^{i}-e_{\alpha}^{a} \tilde{e}_{a}^{j^{\prime}} \delta_{j^{\prime}}^{i}
$$

This structure is independent of the fact we have chosen the first $r$ components of Eq.(2) to solve for $\dot{x}_{a}$. Any other choice would give equivalent results since we are solving algebraic equations and the constraints are also algebraic.

The canonical Hamiltonian can then be found

$$
H_{0}=P_{i} \dot{x}^{i}-L=\frac{1}{2 m} \tilde{g}^{i^{\prime} j^{\prime}} P_{i^{\prime}} P_{j^{\prime}}
$$

where $\tilde{g}^{i^{\prime} j^{\prime}}=\tilde{e}_{a}^{i^{\prime}} \tilde{e}_{b}^{j^{\prime}} h^{a b}$ is a square $r \times r$ matrix. The Hamiltonian is then

$$
H=H_{0}+\lambda^{\alpha} \phi_{\alpha}
$$

where $\lambda^{\alpha}$ are the Lagrange multipliers which implement the constraints $\phi_{\alpha}$. 
The Poisson algebra of the constraints is found to be

$$
\begin{aligned}
\left\{\phi_{\alpha}, \phi_{\beta}\right\} & =M_{\alpha \beta}, \\
M_{\alpha \beta} & =M_{\alpha \beta}^{i^{\prime}} P_{i^{\prime}}, \\
M_{\alpha \beta}^{i^{\prime}} & =\partial_{[\alpha}\left(e_{\beta]}^{a} \tilde{e}_{a}^{i \prime}\right)-e_{[\alpha}^{a} \tilde{e}_{a}^{j^{\prime}} \partial_{j^{\prime}}\left(e_{\beta]}^{b} \tilde{e}_{b}^{i \prime}\right),
\end{aligned}
$$

and the time evolution of the constraints give the consistency conditions

$$
\begin{aligned}
& N_{\alpha}+M_{\alpha \beta} \lambda^{\beta}=0, \\
& N_{\alpha}=N_{\alpha}^{i^{\prime} j^{\prime}} P_{i^{\prime}} P_{j^{\prime}}, \\
& N_{\alpha}^{i^{\prime} j^{\prime}}=\frac{1}{2 m}\left[-\partial_{\alpha} \tilde{g}^{i^{\prime} j^{\prime}}+e_{\alpha}^{a} \tilde{e}_{a}^{k^{\prime}} \partial_{k^{\prime}} \tilde{g}^{i^{\prime} j^{\prime}}-\tilde{g}^{k^{\prime}\left(i^{\prime}\right.} \partial_{k^{\prime}}\left(e_{\alpha}^{a} \tilde{e}_{a}^{\left.j^{\prime}\right)}\right)\right] .
\end{aligned}
$$

It should be noticed that the above expression can not be written as a linear combination of the constraints $\phi_{\alpha}$ since no $P_{\alpha}$ appears in it.

In order to perform the analysis of the consistency condition Eq.(12) it will be useful to consider first the situation where we recover the usual interpretation for a degenerate metric as a space of lower dimensionality. Then we will proceed to the general case.

\section{A. Locally Integrable Case}

Let us first consider the situation where $e_{i}^{a}$ is locally integrable, that is, it satisfies $\partial_{[i} e_{j]}^{a}=$ 0 everywhere. Then we can easily find that the line element reduces to $d s^{2}=d x^{a} d x^{b} h_{a b}$ showing that the $x^{a}$ are the coordinates of an $r$-dimensional space.

We then find from Eq.(11) that $M_{\alpha \beta}=0$ and from Eq.(14) that $N_{\alpha}=0$ so that all constraints are first class. The local symmetry generated by the constraints $\phi_{\alpha}$ is found in the usual way

$$
\begin{aligned}
& \delta x^{i^{\prime}}=\epsilon^{\alpha}\left\{\phi_{\alpha}, x^{i^{\prime}}\right\}=\epsilon^{\alpha} e_{\alpha}^{a} \tilde{e}_{a}^{i^{\prime}}, \\
& \delta x^{\alpha}=-\epsilon^{\alpha}
\end{aligned}
$$

where $\epsilon^{\alpha}$ is the infinitesimal parameter. The Lagrangian can then be written as

$$
L=\frac{1}{2} m \Delta^{a} \Delta^{b} h_{a b}, \quad \Delta^{a}=\tilde{e}_{i^{\prime}}^{a} \dot{x}^{i^{\prime}}+e_{\alpha}^{a} \dot{x}^{\alpha} .
$$

It is easily verified that this Lagrangian is invariant under Eq.(15).

Now using the gauge freedom in Eq.(15) we can gauge away all $x^{\alpha}$ so that we are left with $r$ degrees of freedom $x^{i^{\prime}}$. The Lagrangian Eq.(16) then becomes

$$
L=\frac{1}{2} m \tilde{g}_{i^{\prime} j^{\prime}} \dot{x}^{i^{\prime}} \dot{x}^{j^{\prime}}
$$

describing a particle in geodesic motion in a $r$-dimensional space with a non-degenerate metric $\tilde{g}_{i^{\prime} j^{\prime}}$. 


\section{B. Non-integrable Case}

If $e_{i}^{a}$ is non-integrable then in general $M_{\alpha \beta}$ will be non-vanishing. Then we have to solve Eq.(12) for the Lagrange multipliers $\lambda^{\alpha}$. This involves inverting Eq.(12) and since $M_{\alpha \beta}$ is an $(D-r) \times(D-r)$ antisymmetric matrix the existence of its inverse will depend on $D-r$. So there are two possibilities depending whether $N_{\alpha}$ vanishes or not.

\section{Non-vanishing $N_{\alpha}$}

Let us consider the case when $D-r$ is even. In this case $M_{\alpha \beta}$ in general has an inverse $M^{\alpha \beta}$ so that 円

$$
\lambda^{\alpha}=-M^{\alpha \beta} N_{\beta}
$$

All constraints $\phi_{\alpha}$ are then second class. The number of independent degrees of freedom in configuration space is then $\frac{1}{2}(D+r)$.

The equations of motion for $x^{i}$ are

$$
\begin{aligned}
& \dot{x}^{i^{\prime}}=\frac{1}{m} \tilde{g}^{i^{\prime} j^{\prime}} P_{j^{\prime}}-e_{\alpha}^{a} \tilde{e}_{a}^{i^{\prime}} \lambda^{\alpha}, \\
& \dot{x}^{\alpha}=\lambda^{\alpha},
\end{aligned}
$$

while for $P^{i^{\prime}}$ we get

$$
\dot{P}_{i^{\prime}}=-\frac{1}{2 m} \partial_{i^{\prime}} \tilde{g}^{j^{\prime} k^{\prime}} P_{j^{\prime}} P_{k^{\prime}}-\partial_{i^{\prime}}\left(e_{\alpha}^{a} \tilde{e}_{a}^{j^{\prime}}\right) P_{j^{\prime}} \lambda^{\alpha}
$$

The Lagrangian can be written as

$$
L=P_{i^{\prime}} \dot{x}^{i^{\prime}}-\frac{1}{2 m} \tilde{g}^{i^{\prime} j^{\prime}} P_{i^{\prime}} P_{j^{\prime}}+P_{i^{\prime}} e_{\alpha}^{a} \tilde{e}_{a}^{i^{\prime}} \dot{x}^{\alpha},
$$

after using the fact that $\phi_{\alpha}$ is a second class constraint. The first two terms of the Lagrangian describes the motion of a particle in a $r$-dimensional space with metric $\tilde{g}_{i^{\prime} j^{\prime}}$. However $\tilde{g}$ depends on $x^{\alpha}$ but its momentum $P_{i^{\prime}} e_{\alpha}^{a} \tilde{e}_{a}^{i^{\prime}}$ is not independent. Therefore the phase space has $2 r+(D-r)=D+r$ dimensions. There is a geodesic motion in $r$ dimensions and in the remaining dimensions the motion is constrained by the motion that takes place in $r$ dimensions.

Let us now consider the situation when $D-r$ is odd. Now $M_{\alpha \beta}$ has no inverse since $\operatorname{det} M=0$. Also the number of constraints $\phi_{\alpha}$ is odd which means that either there are first class constraints among the $\phi_{\alpha}$ or there are new constraints. Indeed since Eq.(12) can not be solved for all $\lambda^{\alpha}$ it means that there is a relation among $N_{\alpha}$ and $M_{\alpha \beta}$ not involving

\footnotetext{
${ }^{1}$ We assume that $M$ has no null eigenvalues. If they exist further constraints will appear. The structure of the new constraints will depend explicitly on the form of $e_{i}^{a}$ and then a general classification is no longer possible.
} 
$\lambda^{\alpha}$. Since Eq.(12) comprises just algebraic equations we can take anyone of them as the new constraint. Let us choose the last component of Eq.(12) as the new constraint $\chi$. Then we can solve the first $D-r-1$ components of Eq.(12) for the first $D-r-1$ components of $\lambda^{\alpha}$

$$
\lambda^{\beta^{\prime}}=-M^{\alpha^{\prime} \beta^{\prime}}\left(N_{\alpha^{\prime}}+M_{\alpha^{\prime}, D-r} \lambda^{D-r}\right), \quad \alpha^{\prime}, \beta^{\prime}=1, \ldots, D-r-1,
$$

assuming that $M_{\alpha^{\prime} \beta^{\prime}}$ has an inverse (see footnote 1). The new constraint is then

$$
\chi=\dot{\phi}_{D-r}=N_{D-r}-M^{\alpha^{\prime} \beta^{\prime}} M_{\alpha^{\prime}, D-r} N_{\beta^{\prime}},
$$

where use was made of Eq.(22). No dependence of $\lambda^{D-r}$ is left as it should be.

We also find the Poisson algebra for the new constraint

$$
\left\{\phi_{\alpha}, \chi\right\}=\frac{\partial \chi}{\partial P_{i^{\prime}}} \partial_{i^{\prime}} \phi_{\alpha}-\frac{\partial \phi_{\alpha}}{\partial P_{\beta}} \partial_{\beta} \chi-\frac{\partial \phi_{\alpha}}{\partial P_{i^{\prime}}} \partial_{i^{\prime}} \chi,
$$

which in general is non-vanishing indicating that the new constraint is second class. The Hamiltonian is now

$$
H=H_{0}+\lambda^{\alpha} \phi_{\alpha}+\mu \chi,
$$

where $\mu$ is a new Lagrange multiplier. The consistency conditions are now

$$
\begin{aligned}
\dot{\chi} & =\left\{\chi, H_{0}\right\}+\lambda^{\alpha^{\prime}}\left\{\chi, \phi_{\alpha^{\prime}}\right\}+\lambda^{D-r}\left\{\chi, \phi_{D-r}\right\}=0, \\
\dot{\phi}_{\alpha^{\prime}} & =N_{\alpha^{\prime}}+\lambda^{\beta^{\prime}} M_{\alpha^{\prime} \beta^{\prime}}+\lambda^{D-r} M_{\alpha^{\prime} D-r}+\mu\left\{\phi_{\alpha^{\prime}}, \chi\right\}=0, \\
\dot{\phi}_{D-r} & =N_{D-r}+\lambda^{\beta^{\prime}} M_{D-r \beta^{\prime}}+\mu\left\{\phi_{D-r}, \chi\right\}=0 .
\end{aligned}
$$

As before, from Eq.(27) we find $\lambda^{\alpha^{\prime}}$

$$
\lambda^{\beta^{\prime}}=-M^{\alpha^{\prime} \beta^{\prime}}\left(N_{\alpha^{\prime}}+\lambda^{D-r} M_{\alpha^{\prime} D-r}+\mu\left\{\phi_{\alpha^{\prime}}, \chi\right\}\right) .
$$

Then Eq.(28) can be rewritten as

$$
\chi+\mu\left(M^{\alpha^{\prime} \beta^{\prime}}\left\{\phi_{\alpha^{\prime}}, \chi\right\} M_{D-r \beta^{\prime}}+\left\{\phi_{D-r}, \chi\right\}\right)=0,
$$

which on the constraint surface gives $\mu=0$. Finally, Eq. (26) allows us to determine $\lambda^{D-r}$

$$
\lambda^{D-r}=-\frac{\left\{\chi, H_{0}\right\}+M^{\alpha^{\prime} \beta^{\prime}} N_{\alpha^{\prime}}\left\{\chi, \phi_{\beta^{\prime}}\right\}}{\left\{\chi, \phi_{D-r}\right\}-M^{\alpha^{\prime} \beta^{\prime}} M_{\alpha^{\prime} D-r}\left\{\chi, \phi_{\beta^{\prime}}\right\}} .
$$

Then all Lagrange multipliers are determined $[$ which means that all constraints are second class. Therefore we have $\frac{1}{2}(D+r-1)$ independent degrees of freedom in configuration space. The dynamics is similar to the former case. It describes the motion of a particle in an $r$-dimensional metric space with metric $\tilde{g}$ with the momentum being constrained by $\phi_{\alpha}$ and $\chi$ so that we have a phase space with $2 r+(D-r-1)=D+r-1$ dimensions.

\footnotetext{
${ }^{2}$ This solution is good if $\left\{\chi, \phi_{D-r}\right\}+M^{\alpha^{\prime} \beta^{\prime}} N_{\alpha^{\prime}} \neq 0$. It is very hard to handle this condition since it involves explicitly the inverse of $M_{\alpha^{\prime} \beta^{\prime}}$. In all cases we verified this condition is true but we do not have a general proof of that. If it fails then new constraints would be possible.
} 


\section{Vanishing $N_{\alpha}$}

When solving Eq.(12) another possibility has to be taken into account. If the degenerate metric is such that $N_{\alpha}^{i^{\prime} j^{\prime}}$ vanishes a little algebra shows that

$$
\partial_{[\alpha} \tilde{e}_{\left.k^{\prime}\right]}^{c}+e_{\alpha}^{a} \tilde{e}_{a}^{l^{\prime}} \partial_{\left[k^{\prime}\right.} \tilde{e}_{\left.l^{\prime}\right]}^{c}=0
$$

and

$$
M_{\alpha \beta}^{i^{\prime}}=\left(\partial_{[\alpha} e_{\beta]}^{c}-e_{[\alpha}^{a} e_{\beta]}^{b} \tilde{e}_{a}^{j^{\prime}} \tilde{e}_{b}^{k^{\prime}} \partial_{j^{\prime}} \tilde{e}_{k^{\prime}}^{c}\right) \tilde{e}_{c}^{i^{\prime}}
$$

Then we have to solve $M_{\alpha \beta} \lambda^{\beta}=0$ with $M_{\alpha \beta}$ given above.

Again we have to consider the cases $D-r$ even and odd separately. If $D-r$ is even in general $M_{\alpha \beta}$ has an inverse (see footnote 1) and all Lagrange multipliers can be determined $\lambda^{\alpha}=0$. All constraints are second class and in configuration space we find $(D+r) / 2$ degrees of freedom. The equations of motion for $x^{i}$ are

$$
\begin{aligned}
\dot{x}^{i^{\prime}} & =\frac{1}{m} \tilde{g}^{i^{\prime} j^{\prime}} P_{j^{\prime}}, \\
\dot{x}^{\alpha} & =0
\end{aligned}
$$

while for $P_{i^{\prime}}$ we obtain

$$
\dot{P}_{i^{\prime}}=-\frac{1}{2 m} \partial_{i^{\prime}} \tilde{g}^{j^{\prime} k^{\prime}} P_{j^{\prime}} P_{k^{\prime}}
$$

The independent degrees of freedom are then $x^{i^{\prime}}$ and $P_{i^{\prime}}$.

For $D-r$ odd again $M_{\alpha \beta}$ has no inverse so some constraints are first class or there must exist new constraints. Following the same procedure as before we can solve $M_{\alpha \beta} \lambda^{\beta}=0$ for $D-r-1$ Lagrange multipliers $\lambda^{\alpha^{\prime}}\left(\alpha^{\prime}, \beta^{\prime}=1 \ldots D-r-1\right)$ leaving $\lambda^{D-r}$ undetermined

$$
\lambda^{\alpha^{\prime}}=-M^{\alpha^{\prime} \beta^{\prime}} M_{\alpha^{\prime} D-r} \lambda^{D-r} .
$$

This means that there must exist one first class constraint which is given by

$$
\Gamma=\phi_{D-r}-M_{\alpha^{\prime} D-r} M^{\alpha^{\prime} \beta^{\prime}} \phi_{\beta^{\prime}}
$$

Therefore there are $D-r-1$ second class constraints $\phi_{\alpha^{\prime}}$ and one first class constraint $\Gamma$ and consistency gives no more constraints. Then we have $2 D-(D-r-1)-2=D+r-1$ degrees of freedom in phase space.

\section{THE RELATIVISTIC PARTICLE}

We will work in a space-time with signature $(-,+,+, \ldots)$. To avoid problems with the interpretation of a degenerate metric in a time-like coordinate we will consider only degeneracy in space-like surfaces. Then the world-line of the particle will be well defined. So we will consider a degenerate metric of minimal rank of 2 . 
The action for a relativistic particle of mass $m$ in a gravitational background is described in the first order formalism by

$$
S=\int d t\left(\frac{1}{2 e} g_{\mu \nu} \dot{x}^{\mu} \dot{x}^{\nu}-\frac{e}{2} m^{2}\right)
$$

where $e$ is the einbein. The canonical momenta to $e$ and $x^{\mu}$ are, respectively,

$$
\Pi_{e}=\frac{\partial L}{\partial \dot{e}}=0, \quad P_{\mu}=\frac{\partial L}{\partial \dot{x}^{\mu}}=e^{-1} g_{\mu \nu} \dot{x}^{\nu} .
$$

Assuming that the metric is not degenerate we find only one constraint $\Pi_{e}=0$. The canonical Hamiltonian is easily found to be

$$
H_{c}=\frac{e}{2}\left(P_{\mu} P_{\nu} g^{\mu \nu}+m^{2}\right)
$$

and consistency leads to the usual constraint $P^{2}+m^{2}=0$. Both constraints are first class and the canonical Hamiltonian vanishes as a consequence of the reparametrization invariance of the action.

When the metric is degenerate we can not invert the velocities in terms of the momenta. This means that new primary constraints will appear. So let us consider again a metric of the form $g_{\mu \nu}=e_{\mu}^{a^{\prime}} e_{\nu}^{b^{\prime}} \eta_{a^{\prime} b^{\prime}}$ where $\eta_{a^{\prime} b^{\prime}}=\operatorname{diag}(-1,+1, \ldots,+1)$, and $\mu, \nu=0,1, \ldots, D-1$, $a^{\prime}, b^{\prime}=0,1, \ldots, r-1$. It is easily verified that the determinant of this metric vanishes and that it has rank $r$. Let us split the range of the curved space indices into $\mu^{\prime}, \nu^{\prime}=0,1, \ldots, r-1$ and $\underline{\mu}, \underline{\nu}=r, \ldots, D-1$. With this choice for the metric there is an additional primary constraint. It is given by

$$
\Phi_{\underline{\mu}}=P_{\underline{\mu}}-e_{\underline{\mu}}^{a^{\prime}} \tilde{e}_{a^{\prime}}^{\nu^{\prime}} P_{\nu^{\prime}}
$$

and satisfies the Poisson bracket algebra

$$
\left\{\Phi_{\underline{\mu}}, \Phi_{\underline{\nu}}\right\}=M_{\underline{\mu} \underline{\nu}}
$$

where

$$
\begin{aligned}
M_{\underline{\mu} \underline{\underline{\nu}}} & =P_{\lambda^{\prime}}\left(\partial_{[\underline{\mu}} h_{\underline{\nu}]}^{\lambda^{\prime}}-h_{[\underline{\mu}}^{\rho^{\prime}} \partial_{\rho^{\prime}} h_{\underline{\nu}]}^{\lambda^{\prime}}\right), \\
N_{\underline{\mu}} & =e \tilde{P}_{a^{\prime}} \tilde{P}_{d^{\prime}} \tilde{e}_{c^{\prime}}^{\chi^{\prime}} \eta^{c^{\prime} d^{\prime}}\left(\partial_{[\underline{\mu}} e_{\left.\chi^{\prime}\right]}^{a^{\prime}}-h_{\underline{\mu}}^{\lambda^{\prime}} \partial_{\left[\lambda^{\prime}\right.} e_{\left.\chi^{\prime}\right]}^{a^{\prime}}\right),
\end{aligned}
$$

and $h_{\underline{\mu}}^{\lambda^{\prime}}=e_{\underline{\mu}}^{a^{\prime}} \tilde{e}_{a^{\prime}}^{\lambda^{\prime}}$. The canonical Hamiltonian is given by

$$
H_{c}=\frac{e}{2}\left(\tilde{P}^{2}+m^{2}\right)
$$

where

$$
\tilde{P}^{2}=\tilde{g}^{\mu^{\prime} \nu^{\prime}} P_{\mu^{\prime}} P_{\nu^{\prime}}, \quad \tilde{g}^{\mu^{\prime} \nu^{\prime}}=\eta^{a^{\prime} b^{\prime}} \tilde{e}_{a^{\prime}}^{\mu^{\prime}} \tilde{e}_{b^{\prime}}^{\nu^{\prime}}
$$

It follows that the the primary Hamiltonian is 


$$
H_{p}=\frac{e}{2}\left(\tilde{P}^{2}+m^{2}\right)+\lambda \Pi_{e}+\lambda \underline{\underline{\mu}} \Phi_{\underline{\mu}},
$$

where $\lambda$ and $\lambda^{\underline{\mu}}$ are Lagrange multipliers for the primary constraints.

The consistency of the primary constraints result in the secondary constraint

$$
\varphi=-\frac{1}{2}\left(\tilde{P}^{2}+m^{2}\right),
$$

and the consistency condition

$$
N_{\underline{\mu}}+\lambda \underline{\underline{\nu}} M_{\underline{\mu}} \underline{\underline{\nu}}=0 .
$$

Notice that the consistency of $\Pi_{e}$ results in a constraint analogous to usual case with the important difference that $\tilde{P}$ which appears in it and has a form which is similar to that of the non-relativistic case.

Assuming that $M_{\underline{\mu} \underline{\underline{\mu}}}$ and $N_{\underline{\mu}}$ do not vanish we can consider, as in the non-relativistic particle case, two situations depending on the parity of $D-r$.

In the case with $D-r$ even the inverse of $M_{\underline{\mu} \underline{\underline{\nu}}}$ there exists (see footnote 1) and we solve Eq.(49) for the Lagrange multiplier

$$
\lambda^{\underline{\nu}}=-N_{\underline{\mu}} M \underline{\mu} \underline{\underline{\nu}} .
$$

There are then $D-r+1$ second class constraints, that is, an odd number of them. This means that there must exist first class constraints among them. To find them let us take the linear combination

$$
\phi=f \varphi+f \underline{\mu} \phi_{\underline{\mu}},
$$

and impose that it is first class. We then find

$$
\begin{aligned}
& \frac{2}{e} f^{\underline{\mu}} N_{\underline{\mu}}=0, \\
& -\frac{2}{e} f N_{\underline{\rho}}+f \underline{\mu} M_{\underline{\mu \rho}}=0 .
\end{aligned}
$$

The solution for these equations are

$$
\begin{aligned}
f^{\underline{\mu}} & =N_{\underline{\underline{\rho}}} M \underline{\mu \rho}, \\
f & =\frac{e^{2}}{2} .
\end{aligned}
$$

Then the first class constraint is given by

$$
\phi=\frac{e}{2} \varphi+M \underline{\mu \rho} N_{\underline{\rho}} \Phi_{\underline{\mu}} .
$$

Therefore there are $D-r$ second class constraints $\Phi_{\underline{\mu}^{\prime}}$ and one first class constraint $\phi$. This gives $D+r-2$ degrees of freedom in phase space.

In the case of $D-r$ odd there is no inverse for $M_{\underline{\mu \nu}}$. Proceeding as in the non-relativistic case we select the last component Eq.(49) using the notation $\underline{\mu}=\left(\underline{\mu}^{\prime}, \underline{D-r}\right), \underline{\mu}^{\prime}=1, \ldots, D-$ $r-1$. We can then solve for $\lambda \underline{\underline{\nu}}^{\prime}$ finding 


$$
\lambda^{\underline{\nu^{\prime}}}=-M \underline{\underline{\mu}}^{\prime} \underline{\underline{\nu}}^{\prime}\left(N_{\underline{\mu}^{\prime}}+M_{\underline{\mu^{\prime}} \underline{D-r}} \lambda \underline{D-r}\right)
$$

assuming that $M_{\mu^{\prime} \nu^{\prime}}$ has an inverse (see footnote 1). The component $\underline{D-r}$ of Eq(49) gives a new constraint

$$
\chi=N_{\underline{D-r}}-M_{\underline{D-r} \underline{\beta^{\prime}}} M^{\underline{\alpha}^{\prime} \underline{\beta}^{\prime}} N_{\underline{\alpha}^{\prime}} .
$$

The set of constraints is now $\Pi_{e}, \varphi$ and $\chi$. From the time evolution of $\chi$ we get

$$
\lambda \frac{D-r}{D}=-\frac{\frac{e}{2}\{\chi, \varphi\}+M \underline{\rho}^{\prime} \underline{\mu}^{\prime} N_{\underline{\rho}^{\prime}}\left\{\chi, \Phi_{\underline{\mu}^{\prime}}\right\}}{\left(\left\{\chi, \Phi_{\underline{D-r}}\right\}-M \underline{\rho}^{\prime} \underline{\mu}^{\prime} M_{\underline{\rho}^{\prime} \underline{D-r}}\left\{\chi, \Phi_{\underline{\mu}^{\prime}}\right\}\right)} .
$$

To be sure that the denominator of Eq.(59) does no vanish consider the null eigenvector of $M_{\underline{\mu} \underline{\underline{\nu}}}$. It can easily be found to be

$$
V \underline{\mu}=\delta \underline{\underline{\underline{D}-r}}-M_{\underline{D-r}} \underline{\beta}^{\prime} M^{\underline{\beta}^{\prime}} \underline{\underline{\prime}}^{\prime} \delta_{\underline{\underline{\nu}^{\prime}}} .
$$

We then find that the denominator of Eq.(59) can be written as $V \underline{\mu}\left\{\chi, \Phi_{\underline{\mu}}\right\}$ and it is nonvanishing.

We have then found that the Lagrange multipliers $\lambda \underline{\underline{\nu}}$ and $\lambda \underline{D-r}$ are determined. Therefore the constraints $\Phi_{\mu}$ are second class. We have now to find out which class the constraint $\chi$ belongs to. Following the same steps as in the non-relativistic case we consider the extended Hamiltonian

$$
H=\frac{e}{2} \varphi+\lambda \underline{\underline{\mu}} \Phi_{\underline{\mu}}+\mu \chi
$$

Using it to evaluate the time evolution of the constraints we find that

$$
\begin{gathered}
\dot{\varphi}=\lambda \underline{\mu}\left\{\varphi, \Phi_{\underline{\mu}}\right\}+\mu\{\varphi, \chi\} \\
=\frac{-2}{e} \lambda \underline{\mu}^{\prime} N_{\underline{\mu^{\prime}}}-\frac{2}{e} \lambda \underline{D-r} N_{\underline{D-r}}+\mu\{\varphi, \chi\}=0, \\
\dot{\chi}=\frac{e}{2}\{\chi, \varphi\}+\lambda \underline{\mu}\left\{\chi, \Phi_{\underline{\mu}}\right\}=0, \\
\dot{\Phi}_{\underline{\mu}^{\prime}}=N_{\underline{\mu^{\prime}}}+\lambda \underline{\nu}^{\prime} M_{\underline{\mu}^{\prime}} \underline{\underline{\nu}^{\prime}}+\lambda \underline{D-r} M_{\underline{\mu^{\prime}}} \underline{D-r}+\mu\left\{\Phi_{\underline{\mu^{\prime}}}, \chi\right\}=0, \\
\dot{\Phi}_{\underline{D-r}}=N_{\underline{D-r}}+\lambda \underline{\underline{L}}^{\prime} M_{\underline{D-r^{\prime}}} \underline{\nu}^{\prime}+\mu\left\{\Phi_{\underline{D-r}}, \chi\right\}=0 .
\end{gathered}
$$

From Eq.(64) we get

$$
\lambda^{\underline{\alpha}^{\prime}}=-M^{\underline{\alpha}^{\prime}} \underline{\mu}^{\prime}\left(N_{\underline{\mu^{\prime}}}+\lambda \underline{D-r} M_{\underline{\mu^{\prime}} \underline{D-r}}+\mu\left\{\Phi_{\underline{\mu^{\prime}}}, \chi\right\}\right) .
$$

From Eq.(65) we get $\mu=0$ and from Eq.(63) we find again the result already found in Eq.(59). Finally Eq.(65) vanishes identically. Therefore all Lagrange multipliers associated 
to the constraints $\chi$ and $\Phi_{\underline{\mu}}$ are determined showing that they are second class. We also have that

$$
\left\{\varphi, \Phi_{\underline{\mu}}\right\}=-\frac{2}{e} N_{\underline{\mu}} \neq 0,
$$

so that $\varphi$ is also second class. This gives an odd number of second class constraints for $D-r$ odd. This implies then that there must exist a combination of them which is first class.

To find out the first class combination let us denote the set of second class constraints as

$$
\Gamma_{(\xi)}=\left(\varphi, \chi, \Phi_{\underline{\mu}}\right)=\left(\Gamma_{(A)}, \Gamma_{(B)}, \Gamma_{(\underline{\mu})}\right)
$$

and the matrix of the Poisson brackets of $\Gamma_{(\xi)}$ as $\gamma_{(\xi)(\eta)}=\left\{\Gamma_{(\xi)}, \Gamma_{(\eta)}\right\}$ so that $\operatorname{det} \gamma=0$. The null eigenvector of $\gamma$, which satisfies $W^{(\xi)} \gamma_{(\xi)(\eta)}=0$, is then

$$
W^{(\xi)}=\delta_{(D-r)}^{(\xi)}-\gamma_{(D-r)\left(\eta^{\prime}\right)} \gamma^{\left(\eta^{\prime}\right)\left(\zeta^{\prime}\right)} \delta_{\left(\zeta^{\prime}\right)}^{(\xi)}
$$

By imposing that the following linear combination of $\Gamma_{(\xi)}$

$$
\xi=W^{(\xi)} \Gamma_{(\xi)}
$$

is first class results in

$$
\xi=\Phi_{\underline{D-r}}-\left\{\Phi_{\underline{D-r}}, \Gamma_{\left(\eta^{\prime}\right)}\right\} \gamma^{\left(\eta^{\prime}\right)\left(\xi^{\prime}\right)} \Gamma_{\left(\xi^{\prime}\right)} .
$$

We then have the second class constraints $\varphi, \chi$ and $\Phi_{\underline{\mu}}$ and one first class constraint $\xi$ so that there are $D+r-5$ degrees of freedom in phase space.

\section{STRINGS}

The action for a string in a gravitational background $G_{\mu \nu}(x)$ in $D$ dimensions is given by

$$
S=-\frac{1}{4 \pi \alpha^{\prime}} \int d \sigma d \tau \sqrt{-h} h^{(a)(b)}(\sigma, \tau) G_{\mu \nu}(x(\sigma, \tau)) \partial_{(a)} x^{\mu} \partial_{(b)} x^{\nu}
$$

where $h_{(a)(b)}$ is the world-sheet metric, $\mu, \nu=0, \ldots, D-1$ and $(a),(b)=0,1$. The equations of motion are

$$
\begin{aligned}
& \frac{1}{\sqrt{-h}} \partial_{(a)}\left(\sqrt{-h} h^{(a)(b)} \partial_{(b)} x^{\mu}\right) G_{\lambda \mu}+\Gamma_{\mu \nu \lambda} h^{(a)(b)} \partial_{(a)} x^{\mu} \partial_{(b)} x^{\nu}=0, \\
& T_{(a)(b)}=G_{\mu \nu}\left(\partial_{(a)} x^{\mu} \partial_{(b)} x^{\nu}-\frac{1}{2} h_{(a)(b)} h^{\left(a^{\prime}\right)\left(b^{\prime}\right)} \partial_{\left(a^{\prime}\right)} x^{\mu} \partial_{\left(b^{\prime}\right)} x^{\nu}\right)=0,
\end{aligned}
$$

and do not depend on the inverse of $G_{\mu \nu}$. Therefore the action and equations of motion are well defined even in the case of degenerate metrics.

In the orthogonal gauge, $h_{a b}=\eta_{a b}$, Eqs. (73, 74) reduce to 


$$
\begin{gathered}
G_{\lambda \mu} \hat{\partial}_{+} \hat{\partial}_{-} x^{\mu}+\Gamma_{\mu \nu \lambda} \hat{\partial}_{+} x^{\mu} \hat{\partial}_{-} x^{\nu}=0 \\
T_{ \pm \pm}=G_{\mu \nu} \hat{\partial}_{ \pm} x^{\mu} \hat{\partial}_{ \pm} x^{\nu}=0
\end{gathered}
$$

where $\hat{\partial}_{ \pm}=\partial_{\tau} \pm \partial_{\sigma}, \partial_{\tau}=\partial_{0}$ and $\partial_{\sigma}=\partial_{1}$.

As we can notice there are chiral solutions for the equations of motion Eqs. (75, 76) in the form $\hat{\partial}_{+} x^{\mu}=0$ or $\hat{\partial}_{-} x^{\mu}=0$ which are independent of the metric $G_{\mu \nu}$ [13. However we know that the choice of the conformal gauge still leaves a residual symmetry which can be fixed by the gauge choice $x^{+} \equiv \frac{1}{\sqrt{2}}\left(x^{0}+x^{D-1}\right)=\left(x_{0}^{+}+P \tau\right)$, where $P$ is a constant. In this gauge the string coordinate $x^{+}$is no longer chiral, that is $\hat{\partial}_{+} x^{+} \neq 0$. If we insist in having a chiral solution for the other string coordinates we find that only certain gravitational backgrounds admit such solutions [13]. They are given by

$$
\begin{aligned}
& G_{--}=\tilde{g}_{--}, \quad G_{-i}=\tilde{g}_{-i}, \quad G_{+j}=\tilde{g}_{i j}, \\
& G_{+i}=\partial_{i} \tilde{h}, \quad G_{+-}=\partial_{-} \tilde{h}, \quad G_{++}=0,
\end{aligned}
$$

where $\tilde{g}_{--}, \tilde{g}_{-i}, \tilde{g}_{i j}$ and $\tilde{h}$ are functions of $x^{-}$and $x^{i}$. Among these allowed gravitational backgrounds we can find some which give rise to a degenerate metric. The most general form for the metric is given by 13

$$
\begin{aligned}
& G_{+-}=\partial_{-} \tilde{h}, \quad G_{++}=0, \quad G_{-i}=\left[a_{i} x^{+}+g_{i}\left(x^{i}\right)\right] G_{+-}, \\
& G_{+i}=a_{i} \tilde{h}+\partial_{i} \tilde{h}, \quad G_{--}=0, \quad G_{i j}=\frac{\left.G_{+(i} G_{-j}\right)}{G_{+-}}
\end{aligned}
$$

where $a_{i}$ are constants and $g_{i}$ are functions of $x^{j}$. We then find that strings satisfying a chiral condition can propagate in a degenerate gravitational background.

We now develop the Hamiltonian formalism in order to find the constraints when the metric in Eq.(72) is degenerated. Introducing the string tension $T=1 /\left(2 \pi \alpha^{\prime}\right)$ we can find the momenta densities

$$
\begin{gathered}
\mathcal{P}_{(a)(b)}=\frac{\partial L}{\partial \dot{h}^{(a)(b)}}=0 \\
\mathcal{P}_{\mu}=\frac{\partial L}{\partial \dot{x}^{\mu}}=-T \sqrt{-h} h^{0(b)} \partial_{(b)} x^{\nu} G_{\mu \nu}(x) .
\end{gathered}
$$

When the metric is non-degenerate the primary constraints are

$$
\Phi_{(a)(b)}=\mathcal{P}_{(a)(b)},
$$

and the primary Hamiltonian is

$$
H=\int_{0}^{2 \pi} d \sigma\left(\mathcal{H}_{c}+\lambda^{(a)(b)} \Phi_{(a)(b)}\right)
$$

where $\lambda^{(a)(b)}$ are Lagrange multipliers and $\mathcal{H}_{c}$ is the canonical Hamiltonian 


$$
\mathcal{H}_{c}=-\frac{1}{2 h^{00}}\left[\frac{(-h)^{-1 / 2}}{T} P_{\mu} P_{\nu} G^{\mu \nu}+2 h^{01} P_{\mu} x^{\prime \mu}+T(-h)^{-1 / 2} x^{{ }^{\prime}{ }^{\prime}} x^{{ }^{\nu}} G_{\mu \nu}\right]
$$

Consistency of the primary constraints leads to the secondary constraints

$$
\begin{gathered}
\varphi_{1}=\frac{1}{T} P_{\mu} x^{\prime \mu} \\
\varphi_{2}=\frac{1}{2}\left(\frac{1}{T^{2}} P_{\mu} P_{\nu} G^{\mu \nu}+x^{\prime \mu} x^{\prime \nu} G_{\mu \nu}\right) .
\end{gathered}
$$

The secondary constraints do not generate any new constraints. As usual all constraints are first class and close the Virasoro algebra.

Let us now consider a degenerate metric of rank $r$ which can be set in the form $G_{\mu \nu}=$ $e_{\mu}^{a^{\prime}} e_{\nu}^{b^{\prime}} \eta_{a^{\prime} b^{\prime}}$ with $a^{\prime}, b^{\prime}=0,1 \ldots r-1$. As in the relativistic particle case we will consider only degeneracy in the space-like directions. The primary constraint Eq.(82) is still the same but the momenta Eq.(81) can no longer be inverted for the velocities. Following the development of the relativistic particle we split the space-time indices $\mu, \nu$ according to $\mu^{\prime}, \nu^{\prime}=0,1, \ldots, r-1$ and $\underline{\mu}, \underline{\nu}=r, \ldots, D-1$. This allows to define the inverse of $e_{\mu^{\prime}}^{a^{\prime}}$ which is a square matrix as $\tilde{e}_{a^{\prime}}^{\mu^{\prime}}$.

Then, besides the constraint Eq.(82) there are further primary constraints which can be written as

$$
\Phi_{\underline{\mu}}=\mathcal{P}_{\underline{\mu}}-e_{\underline{\mu}}^{a^{\prime}} \tilde{e}_{a^{\prime}}^{\nu^{\prime}} \mathcal{P}_{\nu^{\prime}}
$$

where

$$
\begin{aligned}
\mathcal{P}_{\mu} & =\Delta_{a^{\prime}} e_{\mu}^{a^{\prime}}, \\
\Delta_{a^{\prime}} & =M^{0(b)} \partial_{(b)} x^{\nu} e_{\nu}^{b^{\prime}} \eta_{a^{\prime} b^{\prime}} .
\end{aligned}
$$

Notice that we can write this constraint as

$$
\begin{aligned}
\Phi_{\underline{\mu}} & =\mathcal{U}_{\underline{\mu}}^{\lambda} \mathcal{P}_{\lambda}, \\
\mathcal{U}_{\underline{\mu}}^{\lambda} & =\delta_{\underline{\mu}}^{\lambda}-e_{\underline{\mu}}^{a^{\prime}} \tilde{e}_{a^{\prime}}^{\nu^{\prime}} \delta_{\nu^{\prime}}^{\lambda},
\end{aligned}
$$

showing that the metric $G_{\mu \nu}$ has $D-r$ null eigenvectors such that $\mathcal{U}_{\underline{\mu}}^{\lambda} G_{\lambda \nu}=0$.

The canonical Hamiltonian Eq.(84) can then be rewritten as

$$
\mathcal{H}_{c}=-\frac{1}{2 h^{00}}\left[\frac{(-h)^{-1 / 2}}{T} \tilde{\mathcal{P}}^{2}+2 h^{01} P_{\mu} x^{\prime \mu}+T(-h)^{-1 / 2} x^{\prime 2}\right]
$$

where

$$
\begin{aligned}
\tilde{\mathcal{P}}^{2} & =\tilde{G}^{\mu^{\prime} \nu^{\prime}} \mathcal{P}_{\mu^{\prime}} \mathcal{P}_{\nu^{\prime}}=\eta^{a^{\prime} c^{\prime}} \tilde{e}_{a^{\prime}}^{\mu^{\prime}} \tilde{e}_{c^{\prime}}^{\nu^{\prime}} \mathcal{P}_{\mu^{\prime}} \mathcal{P}_{\nu^{\prime}} \\
x^{\prime 2} & =x^{\prime \mu} x^{\prime \nu} G_{\mu \nu}=x^{\prime \mu} x^{\prime \nu} e_{\mu}^{a^{\prime}} e_{\nu}^{b^{\prime}} \eta_{a^{\prime} b^{\prime}}
\end{aligned}
$$

We now build the primary Hamiltonian which will be used to find the consistency conditions for the primary constraints. It is given by 


$$
H_{p}=\int\left(\mathcal{H}_{c}+\lambda^{(a)(b)} \mathcal{P}_{(a)(b)}+\lambda \underline{\nu}_{\underline{\nu}}\right) d \sigma^{\prime}
$$

where $\lambda^{(a)(b)}$ and $\lambda \underline{\nu}$ are the Lagrange multipliers. We then obtain the secondary constraints

$$
\begin{gathered}
\varphi_{1}=\frac{1}{T} \mathcal{P}_{\mu} x^{\prime \mu}, \\
\varphi_{2}=\frac{1}{2}\left(\frac{\tilde{\mathcal{P}}^{2}}{T^{2}}+x^{\prime 2}\right) .
\end{gathered}
$$

This allows the canonical Hamiltonian Eq.(91) to be rewritten as

$$
\mathcal{H}_{c}=-\frac{T}{h^{00}}\left[(-h)^{-1 / 2} \varphi_{2}+h^{01} \varphi_{1}\right] .
$$

Also the constraint algebra can be obtained. For $\varphi_{1}$ and $\varphi_{2}$ we obtain the usual Virasoro algebra

$$
\begin{aligned}
& \left\{\varphi_{1}(\sigma), \varphi_{1}\left(\sigma^{\prime}\right)\right\}=\left[\varphi_{1}(\sigma)+\varphi_{1}\left(\sigma^{\prime}\right)\right] \partial_{\sigma} \delta\left(\sigma-\sigma^{\prime}\right), \\
& \left\{\varphi_{1}(\sigma), \varphi_{2}\left(\sigma^{\prime}\right)\right\}=\left[\varphi_{2}(\sigma)+\varphi_{2}\left(\sigma^{\prime}\right)\right] \partial_{\sigma} \delta\left(\sigma-\sigma^{\prime}\right), \\
& \left\{\varphi_{2}(\sigma), \varphi_{2}\left(\sigma^{\prime}\right)\right\}=\left[\varphi_{1}(\sigma)+\varphi_{1}\left(\sigma^{\prime}\right)\right] \partial_{\sigma} \delta\left(\sigma-\sigma^{\prime}\right),
\end{aligned}
$$

while for $\Phi_{\underline{\mu}}$ we get

$$
\begin{gathered}
\left\{\Phi_{\underline{\mu}}(\sigma), \Phi_{\underline{\nu}}\left(\sigma^{\prime}\right)\right\}=\mathcal{M}_{\underline{\mu}} \underline{\nu}(\sigma) \delta\left(\sigma-\sigma^{\prime}\right), \\
\left\{\Phi_{\underline{\mu}}(\sigma), \varphi_{1}\left(\sigma^{\prime}\right)\right\}=-\Phi_{\underline{\mu}}\left(\sigma^{\prime}\right) \partial_{\sigma^{\prime}} \delta\left(\sigma-\sigma^{\prime}\right), \\
\left\{\Phi_{\underline{\mu}}(\sigma), \varphi_{2}\left(\sigma^{\prime}\right)\right\}=n_{\underline{\mu}}(\sigma) \delta\left(\sigma-\sigma^{\prime}\right),
\end{gathered}
$$

where

$$
\begin{aligned}
& \mathcal{M}_{\underline{\mu} \underline{\nu}}=\mathcal{P}_{\lambda^{\prime}} \tilde{e}_{b^{\prime}}^{\lambda^{\prime}} \partial_{[\underline{\mu}} e_{\underline{\nu}]}^{b^{\prime}}(\sigma)+\mathcal{P}_{\rho^{\prime}} e_{[\underline{L}}^{b^{\prime}} \partial_{\underline{\mu}]} \tilde{e}_{b^{\prime}}^{\rho^{\prime}}(\sigma) \\
& +\mathcal{P}_{\lambda^{\prime}} \partial_{\rho^{\prime}} \tilde{e}_{a^{\prime}}^{\lambda^{\prime}} e_{[\underline{\mu}}^{a^{\prime}} e_{\underline{\nu}}^{b^{\prime}} \tilde{e}_{b^{\prime}}^{\rho^{\prime}}(\sigma)+\mathcal{P}_{\lambda^{\prime}} \tilde{e}_{a^{\prime}}^{\lambda^{\prime}} \tilde{e}_{b^{\prime}}^{\rho^{\prime}} e_{[\underline{\nu}}^{b^{\prime}} \partial_{\rho^{\prime}} e_{\underline{\mu}]}^{a^{\prime}}(\sigma), \\
& n_{\underline{\mu}}(\sigma)=2 \tilde{\mathcal{P}}_{a^{\prime}} \tilde{\mathcal{P}}_{d^{\prime}} \eta^{c^{\prime} d^{\prime}} \tilde{e}_{c^{\prime}}^{\lambda^{\prime}}(\sigma)\left[\partial_{[\underline{\mu}} e_{\left.\lambda^{\prime}\right]}^{a^{\prime}}(\sigma)-e_{\underline{\mu}}^{b^{\prime}} \tilde{e}_{b^{\prime}}^{\rho^{\prime}} \partial_{\left[\rho^{\prime}\right.} e_{\left.\lambda^{\prime}\right]}^{a^{\prime}}(\sigma)\right] \\
& -U_{\underline{\mu}}^{\beta} \partial_{\beta} G_{\lambda \rho} x^{\prime \lambda} x^{\prime \rho}(\sigma)-2 \partial_{\sigma} U_{\underline{\mu}}^{\nu} G_{\lambda \nu} x^{\prime \lambda}(\sigma) \text {. }
\end{aligned}
$$

Consistency of $\Phi_{\underline{\mu}}$ then requires

$$
\mathcal{N}_{\underline{\nu}}(\sigma)+\lambda \underline{\mu} \mathcal{M}_{\underline{\mu}} \underline{\nu}(\sigma)=0,
$$


where $\mathcal{N}_{\mu}(\sigma)=c(\sigma) n_{\mu}(\sigma)$ and $c(\sigma)=-T /\left(2 \sqrt{-h} h^{00}\right)$. This result is similar to those of the non-relativistic and relativistic particles. Again we have to consider separately the cases in which $D-r$ is even and odd.

Let us assume that $\mathcal{N}_{\underline{\mu}} \neq 0$. For $D-r$ even there exists the inverse of $\mathcal{M}_{\underline{\mu}} \underline{\underline{\nu}}$ (see footnote 1) and Eq.(105) can be solved for the Lagrange multiplier

$$
\lambda^{\underline{\nu}}=-\mathcal{M}^{\underline{\mu}} \underline{\underline{\nu}} \mathcal{N}_{\underline{\mu}} .
$$

We then have a set with an odd number of second class constraints $\Phi_{\underline{\mu}}$ and $\varphi_{2}$. We then take a linear combination of these constraints to select the first class constraint which is mixed with the second class ones. We find that it is given by

$$
\phi=c \varphi_{2}+\mathcal{N}_{\underline{\rho}} \mathcal{M} \underline{\rho} \underline{\mu} \Phi_{\underline{\mu}}
$$

Then we have an even number of second class constraints $\Phi_{\underline{\mu}}$ and the first class constraints $\phi, \varphi_{1}$ and $\mathcal{P}_{(a)(b)}$ resulting in $D+r-4$ degrees of freedom in phase space.

For $D-r$ odd we follow the same steps as in the former cases. Since the determinant of $\mathcal{M}_{\underline{\mu} \underline{\underline{\nu}}}$ vanishes there is a null eigenvector $\mathcal{V}^{\underline{\mu}}$ such that $\mathcal{V}^{\underline{\mu}} \mathcal{M}_{\underline{\mu} \underline{\underline{\nu}}}=0$. We then find the new constraint $\mathcal{V}^{\underline{\mu}} \mathcal{N}_{\underline{\mu}}=0$. The null eigenvector can be obtained so that the new constraint is written as

$$
\chi=\mathcal{N}_{\underline{D-r}}-\mathcal{M}_{\underline{D-r}} \underline{\beta^{\prime}} M \underline{\beta^{\prime}} \underline{\alpha^{\prime}} \mathcal{N}_{\underline{\alpha^{\prime}}} .
$$

We split the last component of $\underline{\mu}$ as $\left(\underline{\mu^{\prime}}, \underline{D-r}\right)$ and from Eq.(105) we get

$$
\lambda \lambda^{\underline{\nu^{\prime}}}=-\mathcal{M}^{\mu^{\prime}} \underline{\underline{\nu^{\prime}}}\left(\mathcal{N}_{\underline{\mu^{\prime}}}+\mathcal{M}_{\underline{\mu^{\prime}} \underline{D-r}} \lambda \underline{D-r}\right) .
$$

Consistency of $\chi$ then allow us to determine

$$
\lambda \frac{D-r}{\underline{D}}=-\frac{\left\{\chi(\sigma), \frac{1}{2} \varphi\left(\sigma^{\prime}\right)\right\}+\mathcal{M} \underline{\rho^{\prime}} \underline{\mu^{\prime}} \mathcal{N}_{\underline{\rho^{\prime}}}\left\{\chi, \Phi_{\underline{\mu^{\prime}}}\right\}}{\mathcal{V}^{\underline{\mu}}\left\{\chi, \Phi_{\underline{\mu}}\right\}},
$$

where

$$
\mathcal{V}^{\underline{\mu}}\left\{\chi, \Phi_{\underline{\mu}}\right\}=\left\{\chi, \Phi_{\underline{D-r}}\right\}-\mathcal{M}_{\underline{\rho^{\prime}}} \underline{D-r} \mathcal{M}^{\underline{\rho}^{\prime}} \underline{\mu^{\prime}}\left\{\chi, \Phi_{\underline{\mu^{\prime}}}\right\}
$$

Therefore the denominator of Eq.(110) is non-vanishing. In this way we have determined all components of the Lagrange multiplier $\lambda^{\underline{\mu}}$ so that $\Phi_{\underline{\mu}}$ is second class.

Since the Hamiltonian has the same form as the relativistic particle Hamiltonian we find that the Lagrange multiplier $\mu$ vanishes. This implies then that $\chi$ is a second class constraint.

We still have to analyze $\left\{\varphi_{1}, \chi\right\}$. A short calculation shows that

$$
\begin{aligned}
\left\{\varphi_{1}, \chi\right\} & =\left\{\mathcal{P}_{\mu} x^{\prime \mu}, \mathcal{V}^{\underline{\mu}} \mathcal{N}_{\underline{\mu}}\right\} \\
& =\left\{\mathcal{P}_{\mu}, \mathcal{V}^{\underline{\nu}}\right\} x^{\prime \mu} \mathcal{N}_{\underline{\nu}}+\left\{x^{\prime \mu}, \mathcal{V}^{\underline{\nu}}\right\} \mathcal{P}_{\mu} \mathcal{N}_{\underline{\nu}}+\left\{\mathcal{P}_{\mu}, \mathcal{N}_{\underline{\nu}}\right\} x^{\prime \mu} \mathcal{V}^{\underline{\nu}}+\left\{x^{\prime \mu}, \mathcal{N}_{\underline{\nu}}\right\} \mathcal{P}_{\mu} \mathcal{V}^{\underline{\nu}}
\end{aligned}
$$

It is not possible to know whether this vanishes or not since it depends explicitly on the metric. So if $\left\{\varphi_{1}, \chi\right\} \neq 0$ we have an even number of second class constraints. In this case 
there are $D+r-3$ degrees of freedom in phase space. If, on the other side, $\left\{\varphi_{1}, \chi\right\}=0$ we have an odd number of second class constraints and again there must be a combination of them which is first class. Since the structure of the constraints and consistency conditions are similar to those of the relativistic particle the first class constraint will have the same form as in Eq.(71). Therefore we have the second class constraints $\Phi_{\mu^{\prime}}, \varphi_{2}$ and $\chi$ and the first class constraints $\varphi_{1}, \mathcal{P}_{(a)(b)}$ and $\xi$. In this case there are again $\bar{D}+r-3$ degrees of freedom in phase space. Therefore the degrees of freedom counting is independent of the value of $\left\{\varphi_{1}, \chi\right\}$.

\section{ISOMETRIES AND FIRST CLASS CONSTRAINTS}

Let us consider the role of isometries when the metric is degenerate. We will start with the non-relativistic particle case. Consider the following transformation

$$
\delta x^{i}=-\epsilon^{\alpha} K_{\alpha}^{i}
$$

where $\epsilon^{\alpha}$ is a constant infinitesimal parameter and $K_{\alpha}, \alpha=1, \ldots, r$ represents a set of $r$ vectors. The Lagrangian Eq.(11) transforms as

$$
\delta L=-\frac{1}{2} m \epsilon^{\alpha}\left(K_{\alpha}^{l} \partial_{l} g_{i j}+g_{l j} \partial_{i} K_{\alpha}^{l}+g_{i l} \partial_{j} K_{\alpha}^{l}\right) \dot{x}^{i} \dot{x}^{j}-m \dot{\epsilon}^{\alpha} K_{\alpha}^{i} g_{i j} \dot{x}^{j}
$$

Then the transformation Eq.(113) will be an invariance of the action if the Lie derivative of the metric vanishes

$$
\left(\mathcal{L}_{K_{\alpha}} g\right)_{i j}=K_{\alpha}^{m} \partial_{m} g_{i j}+\left(\partial_{i} K_{\alpha}^{m}\right) g_{m j}+\left(\partial_{j} K_{\alpha}^{m}\right) g_{i m}=0,
$$

that is, if the $K_{\alpha}$ are Killing vectors. Notice that we do not need the inverse of the metric anywhere so that it applies also to the case of a degenerate metric.

Now let us consider the degenerate metric given by $g_{i j}=e_{i}^{a} e_{j}^{b} h_{a b}$. According to the analysis made in Section प1 it has $D-r$ null eigenvectors $U_{\alpha}^{i}$ which will imply in the $D-r$ constraints $\Phi_{\alpha}=0$. These constraints will be first class if $\partial_{[i} e_{j]}^{a}=0$ and the transformations generated by them Eqs.(15) will leave the Lagrangian Eq.(11) invariant. However if we consider the transformations Eqs.(15) rewritten as

$$
\delta x^{i}=-\epsilon^{\alpha}(t) U_{\alpha}^{i},
$$

then the invariance of the Lagrangian Eq.(11) requires that

$$
U_{\alpha}^{m} \partial_{m} g_{i j}+\left(\partial_{i} U_{\alpha}^{m}\right) g_{m j}+\left(\partial_{j} U_{\alpha}^{m}\right) g_{i m}=0
$$

that is, $\left(\mathcal{L}_{U_{\alpha}} g\right)_{i j}=0$. Therefore the Lagrangian is invariant if there is an isometry of the degenerate metric with the Killing vectors being the null eigenvectors of the metric.

Let us remark that the transformation Eq.(116) is local in contrast to the non-degenerate case where the parameter does not depend on time. This is due to the fact that the transformation Eq.(116) is generated by a first class constraint which do not exist for the nondegenerate case. 
Let us now consider the situation for strings. In the locally integrable case $\partial_{[\mu} e_{\nu]}^{a^{\prime}}=0$ we have from Eqs.(103,104) that

$$
\begin{aligned}
\mathcal{M}_{\underline{\mu} \underline{\nu}}(\sigma) & =0, \\
\mathcal{N}_{\underline{\underline{\nu}}}(\sigma) & =-c\left(\mathcal{U}_{\underline{\nu}}^{\mu} \partial_{\mu} G_{\lambda \rho} x^{\prime \lambda} x^{\prime} \rho(\sigma)+2 \partial_{\sigma} \mathcal{U}_{\underline{\mu}}^{\mu} G_{\lambda \mu} x^{\prime \lambda}(\sigma)\right) .
\end{aligned}
$$

This form of $\mathcal{N}_{\underline{\mu}}$ does not allow all constraints to be first class as discussed in Section 『V. However, if we consider an isometry condition in the form

$$
\mathcal{U}_{\underline{\nu}}^{\mu} \partial_{\mu} G_{\lambda \rho}+G_{\lambda \mu} \partial_{\rho} \mathcal{U}_{\underline{\nu}}^{\mu}+G_{\mu \rho} \partial_{\lambda} \mathcal{U}_{\underline{\nu}}^{\mu}=0
$$

where $\mathcal{U}_{\underline{\underline{\nu}}}$ represents a null eigenvector of $G_{\lambda \mu}$ then $\mathcal{N}_{\underline{\mu}}(\sigma)=0$. Hence $\dot{\Phi}_{\mu}=0$ does not generate new constraints and we have a set of first class constraints given by $\Phi_{\mu}, \varphi_{1}$ e $\varphi_{2}$. Then the theory will have $D-(D-r+2)=r-2$ degrees of freedom in configuration space. This shows that the isometry condition is necessary in order to reduce all constraints to first class ones.

\section{QUANTIZATION OF THE NON-RELATIVISTIC PARTICLE}

In this section we will consider the path integral quantization of the non-relativistic particle, discussed in Section 【1, for the case $D-r$ even. We recall that in this case there are only second class constraints. To deal with then we will use the Senjanovic formulation [18] appropriate to handle second class constraints in the path integral and the BFT conversion method [19] which turns the second class constraints into a set of first class constraints satisfying an Abelian algebra.

In the Senjanovic formulation a delta functional of the second class constraints Eq.(国) is inserted into the measure of the path integral as well as the determinant of their Poisson brackets Eq.(111), that is

$$
Z=\int D x^{i^{\prime}} D x^{\alpha} D P_{i^{\prime}} D P_{\alpha} \operatorname{det}^{\frac{1}{2}} M \delta(\phi) e^{-i S},
$$

where the action is obtained from the Hamiltonian Eq.(10)

$$
S=\int d t\left(P_{i^{\prime}} \dot{x}^{i^{\prime}}+P_{\alpha} \dot{x}^{\alpha}-\frac{1}{2 m} \tilde{g}^{i^{\prime} j^{\prime}} P_{i^{\prime}} P_{j^{\prime}}\right) .
$$

We can now perform the integral in $P_{\alpha}$ by using the delta functional. We can also exponentiate the determinant by introducing ghost variables $\eta^{\alpha}$. The resulting action is quadratic in $P_{i^{\prime}}$ so that it can also be integrated out. The final effective action is then

$$
S_{e f f}=-\frac{m}{2} \int d t \tilde{g}_{i^{\prime} j^{\prime}}\left(\dot{x}^{i^{\prime}}+\dot{x}^{\alpha} e_{\alpha}^{a} \tilde{e}_{a}^{i^{\prime}}-\eta^{\alpha} M_{\alpha \beta}^{i^{\prime}} \eta^{\beta}\right)\left(\dot{x}^{j^{\prime}}+\dot{x}^{\beta} e_{\beta}^{a} \tilde{e}_{a}^{\tilde{j}^{\prime}}-\eta^{\gamma} M_{\gamma \delta}^{j^{\prime}} \eta^{\delta}\right) .
$$

When performing the last functional integral we get a further determinant in the measure of the path integral

$$
Z=\int D x^{i^{\prime}} D x^{\alpha} D \eta^{\alpha} \operatorname{det}^{\frac{1}{2}} \tilde{g} e^{-i S_{e f f}},
$$


and this determinant is a functional of $x^{i^{\prime}}$ and $x^{\alpha}$ so that the coordinates get mixed up and the final form Eq.(124) is not very useful. Maybe there is a geometrical interpretation for the effective action Eq.(123) but it is not apparent in this form. This of course deserves further investigation. We still have the right degrees of freedom: $r$ from $x^{i^{\prime}}, D-r$ from $x^{\alpha}$ and $-\frac{1}{2}(D-r)$ from the ghosts $\eta^{\alpha}$ adding up to $\frac{1}{2}(D-r)$.

We now turn to the Abelian BFT conversion method. The details of the method can be found in the original papers [19]. To convert the second class constraints into first class ones we need to introduce new variables satisfying certain conditions. To each second class constraint $\phi_{\alpha}(t)$ we associate a new variable $\chi_{\alpha}(t)$ which has vanishing Poisson bracket with all other variables and the constraints, and has non-vanishing Poisson brackets among themselves

$$
\left\{\chi_{\alpha}(t), \chi_{\beta}\left(t^{\prime}\right)\right\}=\omega_{\alpha \beta} \delta\left(t-t^{\prime}\right),
$$

where $\omega_{\alpha \beta}$ is antisymmetric in its indices. We also introduce new first class constraints denoted by $\tilde{\phi}_{\alpha}$ such that

$$
\tilde{\phi}_{\alpha}(t)=\phi_{\alpha}(t)+\sum_{n=1}^{\infty} \tilde{\phi}_{\alpha}^{(n)}(t) .
$$

If we assume that the first term in the sum is of the type

$$
\tilde{\phi}_{\alpha}^{(1)}(t)=\int d t^{\prime} X\left(t-t^{\prime}\right) \chi_{\alpha}\left(t^{\prime}\right)
$$

and that the new constraints form an Abelian algebra we readily find that $X\left(t-t^{\prime}\right)=\delta\left(t-t^{\prime}\right)$, $\omega_{\alpha \beta}=-M_{\alpha \beta}$ and no higher order terms are needed in Eq.(126). Then

$$
\begin{aligned}
\left\{\chi_{\alpha}, \chi_{\beta}\right\} & =-M_{\alpha \beta}, \\
\tilde{\phi}_{\alpha} & =\phi_{\alpha}+\chi_{\alpha} .
\end{aligned}
$$

We can now introduce physical variables $\tilde{x}^{i}, \tilde{P}_{i}$ which have vanishing Poisson brackets with the first class constraints $\tilde{\phi}_{\alpha}$. Assuming an expansion like Eq.(126) for each variable we find that only the first correction is needed. The final result is

$$
\begin{aligned}
& \tilde{x}^{i^{\prime}}=x^{i^{\prime}}+\chi_{\alpha} M^{\alpha \beta} e_{\beta}^{a} \tilde{e}_{a}^{i^{\prime}}, \\
& \tilde{x}^{\alpha}=x^{\alpha}+M^{\alpha \beta} \chi_{\beta}, \\
& \tilde{P}_{i^{\prime}}=P_{i^{\prime}}+\chi_{\alpha} M^{\alpha \beta} \partial_{i^{\prime}}\left(e_{\beta}^{a} \tilde{e}_{a}^{j^{\prime}}\right) P_{j^{\prime}}, \\
& \tilde{P}_{\alpha}=P_{\alpha}+\chi_{\beta} M^{\beta \gamma} \partial_{\alpha}\left(e_{\gamma}^{a} \tilde{e}_{a}^{j^{\prime}}\right) P_{j^{\prime}} .
\end{aligned}
$$

The first class Hamiltonian is then easily found to be

$$
\begin{aligned}
\tilde{H}_{c}\left(x^{i}, P_{i}, \chi_{\alpha}\right) & =H_{c}\left(\tilde{x}^{i}, \tilde{P}_{i}\right) \\
& =\frac{1}{2 m} \tilde{g}^{i^{\prime} j^{\prime}}\left(\tilde{x}^{i}\right)\left(P_{i^{\prime}}+\chi_{\alpha} M^{\alpha \beta} \partial_{i^{\prime}}\left(e_{\beta}^{a} \tilde{e}_{a}^{k^{\prime}}\right) P_{k^{\prime}}\right)\left(P_{j^{\prime}}+\chi_{\alpha} M^{\alpha \beta} \partial_{j^{\prime}}\left(e_{\beta}^{a} \tilde{e}_{a}^{k^{\prime}}\right) P_{k^{\prime}}\right),
\end{aligned}
$$

and the Lagrangian in first order form reads 


$$
L=P_{i} \dot{x}^{i}+\frac{1}{2} \dot{\chi}_{\alpha} M^{\alpha \beta} \chi_{\beta}-\tilde{H}_{c}-\lambda^{\alpha} \tilde{\phi}_{\alpha}
$$

The first class constraints give rise to gauge transformations which are found by computing the Poisson brackets of the variables with the constraints. The gauge transformations which leave the Lagrangian Eq.(131) invariant are

$$
\begin{gathered}
\delta \tilde{x}^{i}=\delta \tilde{P}_{i}=0, \\
\delta \chi_{\alpha}=-\Lambda^{\beta} M_{\alpha \beta},
\end{gathered}
$$

where $\Lambda^{\alpha}(t)$ is the gauge parameter. As expected $\tilde{x}^{i}$ and $\tilde{P}_{i}$ are gauge invariant and only the newly introduced variables are not gauge invariant.

Then we were able to transform the second class constraints into a set of Abelian first class constraints by adding a new variable $\chi_{\alpha}$ which obeys first order equations of motion and is pure gauge. We can find physical variables $\tilde{x}^{i}$ and $\tilde{P}_{i}$ but the resulting Hamiltonian and Lagrangian mix the new and old variables. It was not possible to find a Lagrangian or Hamiltonian solely in terms of the new variables.

\section{CONCLUSIONS}

We have analyzed the constraint structure for relativistic and non-relativistic particles and strings in a background described by a degenerate metric. We have shown that there is a rich structure behind such systems and that the resulting dynamics is non-trivial. A common feature of all cases is the consistency condition of the form $N_{\alpha}+M_{\alpha \beta} \lambda^{\beta}=0$. It allow us to repeat the same procedures in the classification of the constraints in all cases.

We have performed a complete classification of the constraints for the non-relativistic particle. When $e_{\mu}^{a}$ is locally integrable all constraints are first class and the particle has $r$ degrees of freedom in configuration space. An effective Lagrangian can be derived in this case. On the other side, when $e_{\mu}^{a}$ is not integrable we can consider two cases depending on whether $N_{\alpha}$ vanishes or not. For non-vanishing $N_{\alpha}$ and $D-r$ even all constraints are second class and there are $\frac{1}{2}(D+r)$ degrees of freedom; if $D-r$ is odd all constraints are still second class but now there are $\frac{1}{2}(D+r+1)$ degrees of freedom. For both cases it is possible to find the effective Lagrangian. In the case of vanishing $N_{\alpha}$ and $D-r$ even all constraints are again second class and the number of degrees of freedom is $\frac{1}{2}(D+r)$; if $D-r$ is odd then all constraints but one are second class and the remaining one is first class. In this case there are $\frac{1}{2}(D+r-1)$ degrees of freedom.

For the relativistic particle the constraint analysis follows the same lines as in the nonrelativistic case. We have analyzed only the $N_{\underline{\mu}} \neq 0$ case. For $D-r$ even there is one first class constraint and the remaining ones are second class and the particle has $\frac{1}{2}(D+r-2)$ degrees of freedom. For $D-r$ odd all constraints are second class and there are $\frac{1}{2}(D+r-5)$ degrees of freedom. The cases where $N_{\mu}$ vanishes can be analyzed in the same way as for the non-relativistic particle.

The same steps can be taken for strings. Again we considered only the case when $\mathcal{N}_{\mu} \neq 0$. For $D-r$ even there are two first class constraints and the remaining ones are second class. There are $\frac{1}{2}(D+r-4)$ degrees of freedom in configuration space. When $D-r$ is odd all constraints are second class and describe $\frac{1}{2}(D+r-3)$ degrees of freedom. 
When $e_{\mu}^{a}$ is locally integrable we can immediately obtain the first class constraints in the case of the relativistic and non-relativistic particles. However the same is not true for the case of strings. At this point the isometries associated to the degenerate metric showed its relevance since it allowed us to obtain the corresponding first class constraints. It is also important to notice that the Killing vectors are the null eigenvectors of the degenerate metric and this must have some geometrical meaning which at moment is beyond our understanding. The isometries of strings in a gravitational background are important for the formulation of dual actions [20]. In this description the action obtained from a transformation of the components of the original metric is classically equivalent to the initial action. The procedure does not make use of the inverse metric and so a degenerate metric can be used to analyze this sort of duality. This is presently under investigation.

We also have done an initial study towards the quantization of systems in a degenerate metric background. We have considered the non-relativistic particle with $D-r$ even so that only second class constraints are present. We have performed the path integral quantization à la Senjanovic and alternatively using the conversion method of BFT which transforms the second class constraints into first class ones. The resulting effective actions are complicated and no geometrical structures can be seen. This shows that more powerful methods of quantization than those employed here are necessary. It also shows that it is necessary to get a deeper geometrical insight for the constraints in order to recast the effective actions in a more compact form. Work in this direction is in progress.

\section{ACKNOWLEDGMENTS}

LAC would like to thank FAPESP for financial help. This work was partially supported by FAPESP and CNPq. 


\section{REFERENCES}

[1] S. W. Hawking and R. Penrose, Proc. Roy. Soc. Lond. A314, 529 (1970); S. W. Hawking and G. F. R. Ellis, The Large Scale Structure of Space-Time (Cambridge, 1973); for a recent discussion of the role of singularities see G. T. Horowitz and R. Myers, Gen. Rel. Grav. 27 (1995) 915; R. H. Brandenberger, "Nonsingular Cosmology and Planck Scale Physics", preprint Brown-HET-985, gr-qc/9503001.

[2] A. Strominger and E. Martinec, Phys. Lett. B379 (1996) 99; C. G. Callan, Jr. and J. M. Maldacena, Nucl. Phys. B472 (1996) 591; G. T. Horowitz and A. Strominger, Phys. Rev. Lett. 77 (1996) 2368.

[3] G. T. Horowitz and S. F. Ross, JHEP 9804 (1998) 015; A. Lawrence and E. Martinec, Class. Quant. Grav. 13 (1996) 63.

[4] G. T.Horowitz and D. Marolf, Class. Quant. Grav. 12 (1995) 2173.

[5] G. T. Horowitz, Class. Quantum Grav. 8, 587 (1991); J. Louko and R. D. Sorkin, Class. Quant. Grav. 14 (1997) 179 and references therein.

[6] A. Chamblin, "Topology and Causal Structure", gr-qc/9509046.

[7] J. C. Baez, Commun. Math. Phys. 193 (1998) 219.

[8] L. M. Sokolowski, Acta Phys. Pol. B6 (1975) 529, ibidem 657; J. Smallwood, J. Math. Phys. 20 (1979) 459.

[9] I. Bengtsson, Class. Quantum Grav. 8, 1847 (1991); T. Jacobson and J. D. Romano, Class. Quantum Grav. 9, L 119 (1992).

[10] I. Bengtsson and T. Jacobson, Class. Quant. Grav. 14 (1997) 3109.

[11] A. Achúcarro and P. Townsend, Phys. Lett B 180, 89 (1986); E. Witten, Nucl. Phys. B 311, 46 (1988); K. Isler and C. Trugenberger, Phys. Rev. Lett. 63, 834 (1989); A. Chamseddine and D. Wyler, Phys. Lett. B228, 75 (1989); D. Cangemi and R. Jackiw, Phys. Rev. Lett. 69, 233 (1992).

[12] E. Witten, Commun.. Math. Phys. 117, 353 (1988).

[13] O. A. Mattos and V. O. Rivelles, Phys. Rev. Lett. 70, 1583 (1993).

[14] R. P. Zaikov, Phys. Lett. B263 (1991) 206.

[15] A. Karlhede and U. Lindström, Class. Quant. Grav. 3 (1986) L73.

[16] U. Lindström and R. von Unge, Phys. Lett. B403 (1997) 233; H. Gustafsson and U. Lindström, Nucl. Phys. B540 (1999) 520.

[17] D. M. Marolf, Class. Quant. Grav. 11 (1994) 239.

[18] P. Senjanovic, Ann. Phys. (NY) 100 (1976) 227.

[19] I. A. Batalin and E. S. Fradkin, Phys. Lett. B180 (1986) 157; Nucl. Phys. B279 (1987) 157; I. A. Batalin and I. V. Tyutin, Intl. J. Mod. Phys. A6 (1991) 3255.

[20] A. Giveon and M. Rocek, "Introduction to Duality", hep-th/9406178; Nucl. Phys. B194 (1994) 173; T. Buscher, Phys. Lett. B194 (1987) 59. 